Short title: Intrusion-based reasoning in depression

\title{
Intrusion-based reasoning and depression: Cross-sectional and prospective relationships
}

David Berle \& Michelle L. Moulds

School of Psychology, The University of New South Wales, Australia.

Address for correspondence:

David Berle

School of Psychology

The University of New South Wales

Sydney NSW 2052

Australia

Ph: +61293853425

Fax: +61293853641

Email: dberle@psy.unsw.edu.au; david.berle@swahs.health.nsw.gov.au 


\begin{abstract}
Intrusion-based reasoning refers to the tendency to form interpretations about oneself or a situation based on the occurrence of a negative intrusive autobiographical memory. Intrusionbased reasoning characterises posttraumatic stress disorder, but has not yet been investigated in depression. We report two studies that aimed to investigate this. In Study 1, both high $(n=42)$ and low $(n=28)$ dysphoric participants demonstrated intrusion-based reasoning. High dysphoric individuals engaged in self-referent intrusion-based reasoning to a greater extent than did low dysphoric participants. In Study 2, there were no significant differences in intrusion-based reasoning between currently depressed $(n=27)$ and non-depressed $(n=51)$ participants, and intrusion-based reasoning did not predict depressive symptoms at six-month follow-up. Interestingly, previously $(n=26)$ but not currently depressed participants $(n=27)$ engaged in intrusion-based reasoning to a greater extent than never-depressed participants $(n=25)$, indicating the possibility that intrusion-based reasoning may serve as a "scar" from previous episodes. The implications of these findings are discussed.
\end{abstract}

Keywords: mental intrusions, depression, intrusion-based reasoning, autobiographical memory, remission. 
Mental intrusions take the form of unwanted, unbidden and uncontrollable thoughts, images or memories (Harvey, Watkins, Mansell, \& Shafran, 2004; Rachman, 1981).

Although the occurrence of intrusions is considered to be almost universal such that both clinical and non-clinical populations report that they experience these involuntary cognitions (Rachman \& de Silva, 1978; Berntsen, 2010), their excess, persistence and level of associated distress is associated with psychopathology (Moulds \& Holmes, 2011). Intrusive autobiographical memories are one specific type of intrusion that has been studied extensively in the clinical literature; primarily in individuals with posttraumatic stress disorder (PTSD). Recent work has also demonstrated that individuals with major depression report the presence of intrusive memories (e.g., Newby \& Moulds, 2011a), and that the extent to which these memories are intrusive and avoided by depressed individuals predicts depression levels 6 months later, controlling for baseline symptoms (Brewin, Reynolds, \& Tata, 1999). In addition, there is overlap in the features of intrusive memories reported by individuals with PTSD and with depression. For example, in both disorders, these memories are rated as highly vivid and are experienced with a here and now quality (Birrer et al., 2007). While intrusive autobiographical memories are not exclusively negative in their content or in the effect that they have on mood states, in clinical disorders, the nature of these memories is most often reflective of disorder specific concerns.

Beyond establishing the presence of intrusive memories in depression, research efforts have been focused on developing an understanding of the cognitive processes that might contribute to the maintenance of intrusions in this disorder (e.g., Williams \& Moulds, 2007a). One such process is the tendency to form negative appraisals about the occurrence of intrusive memories (e.g., having this memory means that I am going crazy). There is abundant evidence from cross-sectional and longitudinal studies that such appraisals of intrusions are associated with PTSD symptom severity and intrusion-related distress 
(Dunmore, Clark, \& Ehlers, 2001). Studies with dysphoric samples have demonstrated that negative interpretations of intrusive memories are associated with the severity of depressive symptoms (Starr \& Moulds, 2006; Williams \& Moulds, 2008). More recently, Newby and Moulds (2010) reported that clinically depressed participants endorsed more negative appraisals of intrusive memories than a never-depressed group, even though the groups did not differ in the extent to which their memories were intrusive or avoided. In a longitudinal study with a mixed sample of community participants who did not receive psychological treatment between baseline and follow-up, negative appraisals of intrusive memories at baseline predicted follow-up depression, controlling for baseline depression symptoms (Newby \& Moulds, 2011b). Taken together, these studies show that - as in PTSD - negative appraisals of intrusive memories play a key role in the persistence of depression.

In a different yet relevant line of research in the PTSD literature, researchers have examined 'intrusion-based reasoning' (Engelhard, van den Hout, Arntz, \& McNally, 2002; Engelhard, Macklin, McNally, van den Hout, \& Arntz, 2001), defined as “inferring danger from the presence of intrusions" (Engelhard et al., 2001, p. 1341). In this respect, intrusionbased reasoning refers to more than a negative appraisal of oneself or a situation: it instead implies a process of thinking whereby intrusive autobiographical memories are given disproportionate influence in the formation of an interpretation. In these studies, participants were presented with a series of scripts that varied according to objective danger (i.e., whether objective danger or objective safety was indicated) and also according to the presence or absence of an intrusive memory about the trauma. For each script, participants were asked to imagine themselves in the given situation and to provide ratings regarding the danger, safety, uncontrollability, anxiety and the "positivity" of the outcome. For example, one of the scripts described the participant driving along during fourth of July celebrations when firecrackers are set off under his or her car. Two of the endings (both "objective safety" version) were as 
follows: The sudden noise makes you angry, then you laugh to yourself and think: "Well, at least I've got a good excuse for being late" (i.e., objective safety and no intrusion); "The sudden noise triggers upsetting thoughts about Vietnam" (i.e., objective safety and intrusion).

Intrusion-based reasoning was considered to occur if participants provided greater ratings of danger for scripts that included an intrusive trauma-relevant memory compared to those that did not, averaged across the objective nature of each situation. The findings indicated that intrusion-based reasoning characterised Vietnam veterans with PTSD more that veterans without PTSD (Engelhard et al., 2001). In a separate study, intrusion-based reasoning predicted both acute PTSD symptoms as well as PTSD symptoms 3.5 months posttrauma (Engelhard et al., 2002).

In PTSD, the most obvious appraisal that might be influenced by intrusion-based reasoning is the perceived danger of a situation. Depression however, lacks a single defining appraisal or interpretative style that defines the disorder. Indeed, Beck's cognitive model of depression (Beck, Rush, Shaw, \& Emery, 1979) highlights the multitude of ways in which depressed individuals negatively appraise themselves, the world and the future. These include concluding that one is a failure or worthless when things go wrong, as well as, in a more general way, perceiving situations as hopeless or as excessively negative or unfortunate.

Investigating intrusion-based reasoning holds promise for extending our understanding of how intrusive memories in depression are interpreted. The aforementioned studies of intrusive memories in depression have demonstrated that depressed individuals endorse more negative appraisals of their intrusive memories. These appraisals were in some cases negative appraisals about the memory itself (e.g., "I should not be having this memory"; Newby \& Moulds, 2010, p. 153) and in others, negative appraisals about the self associated with having the intrusive memory (e.g., "Having this memory means I'm inadequate"; Newby \& Moulds, 2010, p. 153). However, the focus of intrusion-based 
reasoning is partly on the way a person forms interpretations about themselves in a situation when an intrusive memory arises, and partly on the way in which the person makes sense of the situation in which an intrusive memory occurs. In this respect, there may be two broad domains of intrusion-based reasoning: self-referent intrusion-based reasoning, in which interpretations are made about oneself; and non-self-referent intrusion-based reasoning, in which interpretations are made about others or the situation more generally. So far as nonself-referent intrusion-based reasoning is concerned, a person who has an intrusive memory when in a social situation (for example), may conclude that the situation has not gone well, in contrast to a person who does not experience an upsetting intrusive memory at the time.

There are two lines of evidence that give rise to the possibility that intrusion-based reasoning in depression might be particularly elevated for self-referent interpretations. First, there is a substantial literature suggesting that biases in depression are particularly inflated for interpretations about oneself (see Wisco, 2009 for a review). Second, findings from studies on the conceptually similar process of emotional reasoning (where negative interpretations are made on the basis of negatively valenced emotions), suggest that self-referent interpretations might be especially prominent in any intrusion-based reasoning processes in depression (Berle \& Moulds, in press).

Previous studies of intrusive memories in depression have relied upon the appraisal of intrusive memories of a range of negative events. As a result, there is necessarily considerable variability between participants in terms of both the content of intrusions (e.g., specific situations recalled) as well as the frequency of intrusion occurrence. As such, an advantage of adapting Engelhard et al.'s (2001) intrusion-based reasoning procedure to investigate these processes in depression is that the procedure allows the both the content as well as the occurrence of an intrusive memory to be better controlled. Certainly, there may still be variation among participants in the specific ways in which they imagine the intrusive 
memories outlined in each scenario, but the use of scenarios for each participant at least restricts the nature of the intrusive memory to the broad class of event outlined in each scenario (e.g., being excluded from friends). Such control increases our confidence that any differences that emerge between depressed and non-depressed individuals with respect to their interpretations of the self or the situation are driven by the presence or absence of an intrusive memory, rather than by the features of the recalled situation or the frequency of the intrusion in everyday life.

The relationship between intrusion-based reasoning and depression has not yet been investigated. We aimed to determine whether individuals with elevated levels of depressive symptoms demonstrate greater levels of intrusion-based reasoning, and secondly, whether clinically depressed individuals have elevated levels of intrusion-based reasoning. For the first study, we hypothesised that high dysphoric university participants would demonstrate greater levels of intrusion-based reasoning when compared with low dysphoric students. We expected that increased levels of intrusion-based reasoning would be particularly evident for self-referent interpretations.

Our final aim was to determine whether intrusion-based reasoning is independent of similar constructs such as general dysfunctional attitudes about oneself and the world. Given that, as outlined earlier, intrusion-based reasoning is considered to be a reasoning process that involves more than simply negatively appraising oneself or others, we hypothesised that intrusion-based reasoning scores would not be correlated with scores on the Dysfunctional Attitude Scale - Form A (DAS; Weissman \& Beck, 1978), as scores on the DAS are thought to reflect a tendency to engage in negative appraisals rather than intrusion-based reasoning. 


\section{Method}

\section{Participants}

Undergraduate psychology students $(N=70$, mean age $=19.09 ; S D=2.14 ; 42[60 \%]$ female), who received course credit for their participation, were divided into low dysphoric $(\mathrm{BDI}-\mathrm{II} \leq 4)$ and high dysphoric $(\mathrm{BDI}-\mathrm{II} \geq 14)$ groups. The lower cut-off score of 4 was chosen as this has been used in previous studies (e.g., Williams \& Moulds, 2007b). We chose to use a BDI-II score of 14 to define high dysphoric individuals as this corresponds to at least "mild depression" according to the BDI-II manual. Participants who scored in the mid-range of BDI-II scores (i.e., $\geq 5$ and $\leq 13$ ) were excluded and participated in an alternative study.

\section{Self-report measures}

The following self-report measures were presented in randomised order to reduce the possibility of order effects:

The Beck Depression Inventory II (BDI-II; Beck, Steer, \& Brown, 1996) is a wellvalidated 21-item self-report scale that assesses symptoms of depression. Beck et al. reported an internal consistency (Cronbach's alpha) of 0.92 for the BDI-II in a sample of college students. In the present study, the internal consistency values were .91 for the Study 1 sample (the restricted range of BDI-II scores in the Study 1 sample notwithstanding).

The DAS (Weissman \& Beck, 1978) was used to assess beliefs that are considered to contribute to cognitive vulnerability to depression (Kovacs \& Beck, 1978). The DAS consists of 40 -items that are each rated on a 7 -point Likert scale ( $7=$ fully agree; $1=$ fully disagree $)$ with a higher sum score indicating more pathological dysfunctional attitudes. The DAS has good internal consistency in nonclinical samples (Cronbach's $\alpha=.89$ for Form A) and scores on the DAS are positively correlated with BDI-II scores (Nelson, Stern, \& Cicchetti, 1992). Intrusion-based reasoning task 
Following completion of the self-report questionnaires, participants were administered the intrusion-based reasoning task. The intrusion-based reasoning task was adapted from that used in previous studies of intrusion-based reasoning (Engelhard et al., 2001, 2002) so that the content of items were relevant for depression, rather than PTSD.

Each participant was asked to imagine themselves in the situation described in each scenario which was presented with four different endings: one with an objectively neutral ending where an intrusive memory does not occur, one with an objectively neutral ending where an intrusive memory does occur, one with an objectively negative ending where an intrusive memory does not occur, and one with an objectively negative ending where an intrusive memory occurs, for example:

You are speaking to a good friend...

1. ...As you talk, you learn that you haven't been invited to a party that you thought you would have been invited to. You're not that fussed about this. After all, these things happen from time to time. (Objectively neutral and no intrusive memory)

2. ...As you talk, you learn that you haven't been invited to a party that you thought you would have been invited to. As you think about this, an image pops into your mind of when you were excluded from a group in the playground once in primary school. (Objectively neutral and intrusive memory)

3. ...Your friend tells you that another friend of yours has been telling people horrible things about you behind your back. You're not that fussed about this. Most of your other friends won't believe these things and you know these things aren't true. (Objectively saddening and no intrusive memory)

4. ...Your friend tells you that another friend of yours has been telling people horrible things about you behind your back. As you think about this, an image pops into your 
mind of when you were excluded from a group in the playground once in primary school. (Objectively saddening and intrusive memory)

Participants were then asked to provide the following ratings for each scenario on a 1 to 100 visual analogue scale:

1. How unfortunate is this situation?

2. How negative is this situation?

3. How worthless does this situation suggest that you are?

4. How incompetent does this situation suggest that you are?

5. How hopeless is the situation?

6. How controllable is the situation?

Higher scores for these ratings for scenarios that described the occurrence of an intrusive negative autobiographical memory (i.e., a memory of being excluded in the school playground), in contrast to those that did not, were considered to be indicative of intrusionbased reasoning.

The first, second and fifth of these ratings were considered to be non-self-referent ratings in that they pertained to conclusions about the situation, whereas the third and fourth ratings were considered to be self-referent, in that the ratings involved inferences about oneself. The sixth rating, "How controllable is the situation", was included as a filler item and to reduce the risk of carry-over effects of responses from one scenario to the next.

The intrusion-based reasoning scenarios were intermixed with a series of seven other scenarios that formed part of a larger study of emotional reasoning processes (the findings for the emotional reasoning scripts are summarised elsewhere, Berle \& Moulds, in press). All 36 scripts (nine scenarios with four endings each) were administered in a random order except that we ensured that no two script endings from the same scenario were presented consecutively. 
We calculated intrusion-based reasoning (difference) scores as outlined by Engelhard et al. (2001). This involved subtracting the mean ratings of each script that involved an absence of an intrusive memory from the mean ratings of each script that included an intrusion. In other words, intrusion-based reasoning was considered to have occurred whenever the difference score was greater than zero. We averaged these difference scores across the objectively neutral and objectively negative script endings to ensure that intrusionbased reasoning scores were not confounded by the objective nature of the situation. Thus, an intrusion-based reasoning score of greater than zero implies some degree of intrusion-based reasoning in that a participant would be providing more negative ratings for script endings that included an intrusive autobiographical memory (averaged across objectively neutral and objectively negative situations) compared to script endings that did not include an intrusive memory.

\section{Results}

There were 42 participants in the high dysphoric group (i.e., BDI-II $\geq 14$ ) and 28 participants in the low dysphoric group (BDI-II $\leq 4)$. The relatively high number of participants in the high dysphoric group is consistent with previous studies that have used first year psychology students (e.g., Williams \& Moulds, 2008). There were no significant differences between the low and high dysphoric groups in terms of gender composition or age (see Table 1). As could be expected, the high dysphoric group scored significantly higher than the low dysphoric group on the DAS $(t=5.20, d f=68, p<.001,95 \% \mathrm{CI}=49.80$, 22.18).

The internal consistency (Cronbach's alpha) of the three non-self-referent ratings (how unfortunate, how negative and how hopeless the situation is) was 0.86 , and the internal consistency of the two self-referent ratings (how worthless and incompetent the situation 
suggests one is) was 0.94 . We thus report the analyses for non-self-referent and self-referent mean scores, respectively ${ }^{1}$.

Both the non-self-referent and self-referent scores were significantly greater than zero within each of the low and high dysphoric groups (all $p \mathrm{~s}<0.05)$. This indicates that both dysphoric and non-dysphoric individuals engaged in intrusion-based reasoning, as their ratings for situations where an intrusion occurred were more negative than for situations where an intrusion did not occur.

Figure 1 shows the intrusion-based reasoning scores for the low and high dysphoric groups. For the non-self-referent ratings, the high dysphoric group scored greater than the low dysphoric group $(M=22.66, S D=20.16$ vs $M=14.68, S D=15.26$ respectively), however the between-group difference was not statistically significant $(t=1.78, d f=68, p=$ $.08,95 \% \mathrm{CI}=-16.91,0.97)$. For the self-referent ratings, the high dysphoric group scored significantly higher than the low dysphoric group $(M=17.94, S D=20.71$ vs $M=8.00, S D=$ 16.04 respectively; $t=2.15, d f=68, p=.04,95 \% \mathrm{CI}=-19.19,-0.70)$.

We then correlated each of the intrusion-based reasoning scores for each group with the DAS-A. None of the correlations were greater than 0.25 in magnitude, and none were statistically significant.

\section{Discussion}

There are three noteworthy findings from this study. First, both the low and high dysphoric groups demonstrated intrusion-based reasoning. It appears then, that even individuals with very low levels of depressive symptoms tend to rate situations, as well as themselves in those situations, more negatively if an intrusive memory occurs. This finding is broadly consistent with the slightly more negative ratings for intrusion-based scenarios compared to non-intrusion scenarios rated by the control group of the Engelhard et al. (2001) 
study. Second, the high dysphoric group scored significantly higher than the low dysphoric group for self-referent ratings. This finding is in accordance with research that has suggested that in depression, negative interpretations about oneself, as opposed to others or the world, are particularly prominent (Wisco \& Nolen-Hoeksema, 2010). The results for non-selfreferent interpretations are less clear: while the high dysphoric group scored higher than the low dysphoric group, this difference did not reach statistical significance. Third, intrusionbased reasoning scores did not appear to be associated with dysfunctional attitudes. If intrusion-based reasoning is a risk factor for depression, it is likely then to be independent of dysfunctional attitudes, which have otherwise been considered to be a vulnerability factor for depression, especially in the context of stressful life events (Segal et al., 2006).

Previous studies have found that depressed individuals tend to form negative interpretations of intrusive negative autobiographical memories (e.g., Newby \& Moulds, 2010, Williams \& Moulds, 2008). Our study adds to these findings by also suggesting that individuals with high levels of depressive symptoms are likely to form negative interpretations about themselves in a situation if a negative intrusive memory occurs. This suggests that individuals may feel a degree of agency or responsibility for the occurrence of intrusive memories if they form judgements about themselves when such memories arise.

There are several ways in which the findings of this study could be extended. First, the use of a clinical sample would allow us to more confidently form conclusions about the associations between intrusion-based reasoning and depression. In addition, it would provide the opportunity to test whether our findings of greater intrusion-based reasoning for selfreferent interpretations are replicated in a clinical sample. The more extreme depressive symptoms in a clinical sample (as compared to the symptoms reported by the high dysphoric group in the present study) might also mean that non-self-referent ratings could be 
significantly greater in a group of depressed individuals when compared with a nondepressed group.

Aside from currently-depressed individuals, there remain questions about the level of intrusion-based reasoning in individuals with remitted depression. Is intrusion-based reasoning also elevated for such individuals? If this is the case, then intrusion-based reasoning may serve as a vulnerability factor for further depressive episodes, as a scar from previous depressive episodes, or both. Another possibility is that previously-depressed individuals may associate the occurrence of intrusive negative autobiographical memories with the possible onset of another depressive episode (since depressive episodes are often characterised by rumination and reflection on one's past failures; Nolen-Hoeksema, 1991). If this is the case, then compared with individuals who have never been depressed, those who have previously had depressive episodes may remain particularly vigilant for intrusive negative memories and be particularly prone to try and make sense of them if and when they do occur (e.g., "What does having this memory say about me?").

In addition, there are two methodological limitations of Study 1 that could be addressed in a follow-up study that extends this research. One is that the intrusion-based reasoning procedure that we used relied on the ability of participants to imagine themselves in a given situation. Including an index of the extent to which participants engage in imagery and controlling for this in the analysis would increase our confidence that any between-group differences in intrusion-based reasoning are not an artefact of differences in the tendency to use imagery in everyday life. A second limitation is that we were unable to determine the extent to which intrusion-based reasoning reflects a more general deficit of deductive reasoning. Again, Study 1 could be extended by including such a measure and examining whether any between-group differences in intrusion-based reasoning remain when performance on a general deductive reasoning task is controlled for in the analyses. 
The stability of intrusion-based reasoning has also not yet been investigated.

Determining whether intrusion-based reasoning is a stable tendency, or whether it is something that is variable, and perhaps susceptible to current stressors, will be important if approaches are to be developed to ameliorate any negative consequences of this thinking style. Finally, intrusion-based reasoning has been found to predict subsequent PTSD symptoms, although not after controlling for baseline symptoms (Engelhard et al., 2002). The value of intrusion-based reasoning for predicting subsequent depressive symptoms remains unclear however.

\section{Study 2}

The aim of Study 2 was to extend Study 1 by including a clinical sample and by addressing each of the aforementioned limitations. Our hypotheses were as follows. First, we expected that never-depressed, previously-depressed and currently depressed participants would demonstrate intrusion-based reasoning, consistent with the finding from Study 1 that both the high and low dysphoric groups engaged in intrusion-based reasoning. Second, we predicted that currently depressed individuals would demonstrate greater levels of intrusionbased reasoning than non-depressed individuals, particularly for self-referent interpretations. For our third hypothesis, we predicted that, within the non-depressed group, the previously depressed individuals would demonstrate greater levels of both self- and non-self-referent intrusion-based reasoning than the never-depressed individuals.. Fourth, we hypothesised that scores on the intrusion-based reasoning task would be independent of imagery ability and of deductive reasoning ability.

Method 
A community-based sample comprising 93 adults was recruited via advertisements (online and posters) and from an existing online database of community participants who had taken part in previous studies of depression and who had agreed to be contacted about future research.

Each participant was reimbursed at the rate of \$20/hour. Of the 93 consenting participants, 15 were excluded from the present study: due to a current or recent psychotic or hypomanic episode $(n=5)$, English language difficulties $(n=4)$, procedural problems in administering the emotional reasoning task $(n=4)$ or a diagnosis of current dysthymia but no current major depressive disorder $(n=2)$. We excluded the two individuals with dysthymia as we could not confidently classify them as currently, previously or never-depressed. Thus, the final sample comprised 78 participants.

Given that previous studies that compared clinical with non-clinical groups detected large size between group differences in intrusion-based reasoning (Cohen's $f=0.54 ; N=30$; Engelhard et al., 2001) and given the advantages of within-subject manipulation of intrusion based reasoning, we anticipated that our sample size would be sufficient. The statistical power to detect a large-size effect using a one-way ANOVA if the sample was comprised of three equal groups (depressed, never-depressed and previously-depressed) was 0.88 .

\section{Measures}

\section{Structured clinical interview}

Participants were administered the Structured Clinical Interview for DSM-IV Axis I disorders non-patient edition (SCID-I; First, Spitzer, Gibbons, \& Williams, 1996) to determine the presence of current and lifetime DSM-IV disorders and to allocate participants to the depressed or non-depressed groups. Participants were included in the depressed group if they reported symptoms consistent with a current diagnosis of a major depressive episode. All interviews were conducted by a clinical psychologist with 10 years of experience in 
administering diagnostic interviews. To ensure the fidelity of diagnoses, 24 audio recordings of interviews were independently rated by an experienced clinical psychologist. For the diagnosis of current major depressive episode, there was agreement for 22 of the 24 participants $(91.7 \%$ agreement; Cohen's Kappa $=.78)$.

\section{Self-report measures}

The BDI-II was administered. In this sample, the internal consistency (Cronbach's $\alpha$ ) was .92 and the test-retest correlation across the six-month follow-up interval was $r=.55$.

The Spontaneous Use of Imagery scale was also administered (SUIS; Kosslyn, Chabris, Shephard, \& Thompson, 1998). This is a 12-item scale used to identify the extent to which individuals engage in imagery in their day-today activities. It was included in the present study because the responses that participants provide on the intrusion-based reasoning procedure, which require participants to imagine themselves in various situations, could be influenced by individual differences in the use of mental imagery. Items of the SUIS have a high degree of internal consistency (e.g., .98; Reisberg, Pearson, \& Kosslyn, 2003) and are associated with scores on similar scales such as the Vividness of Visual Imagery

Questionnaire (Reisberg et al., 2003).

\section{Intrusion based reasoning task}

The same two intrusion based reasoning scenarios used in Study 1 were again used in Study 2. Given that the self-referent ratings appeared to be particularly good discriminators between the high and low dysphoria groups in Study 1, we decided to replace the ratings of how hopeless and how (un)controllable the situation is with how pathetic and how inadequate the situation suggested that the respondent would be. Thus, the six ratings used for Study 2 were:

1. How unfortunate is this situation?

2. How negative is this situation? 
3. How worthless does this situation suggest that you are?

4. How incompetent does this situation suggest that you are?

5. How pathetic does this situation suggest that you are?

6. How inadequate does this situation suggest that you are?

As in Study 1, the intrusion based reasoning scenarios were interspersed with seven emotional reasoning scenarios that will be reported in a separate paper and the order of script presentation was random except that no two scripts from the same scenario were presented consecutively.

\section{Deductive reasoning task}

To ensure that emotional reasoning tendencies are not simply an artefact of a more general deductive reasoning deficit, we also administered a modified version of the Wason Selection Task (WST; Wason, 1968). The task was modified so that the hypothetical situations had local and age appropriate relevance (e.g., establishing whether one hour of study each night will necessarily lead one to become fluent in a new language). The task was presented on computer and each participant was instructed to select the minimum number of four "cards" (on the screen) to "turn over" to disconfirm the stated rule, with each card indicating either whether the antecedent had occurred (or not) or whether the consequence had occurred (or not). For instance, for the study related item, the correct response would be to "turn over" the cards "Did study for an hour each night" and "Did not become fluent". The task generates scores for verification and falsification, as well as a total score (higher scores indicating better deductive reasoning ability).

\section{Six-month follow-up}

Participants were invited to complete the same measures and intrusion-based reasoning procedure (without the SCID-IV) six months after attending the initial assessment. Forty-seven (60.3\%) participants attended the follow-up assessment a mean of 6.11 months 
(Median $=6, S D=0.76$ ) after the baseline assessment. The reasons for participant attrition were unclear, although a number of participants were international students who returned to their countries of origin during the follow-up interval. Importantly, there were no statistically significant differences at baseline between participants who did and did not attend the six month follow-up in terms of demographic characteristics, SCID-derived co-occurring disorders, self-report questionnaire scores, or intrusion-based reasoning scores.

\section{Data analyses}

For the purpose of testing our hypotheses relating to intrusion-based reasoning at the baseline assessment we divided the overall sample into three groups. The (currently) depressed group ("depressed"; $n=27$ ), the non-depressed, but previously-depressed group ("previously-depressed"; $n=26$ ), and the non-depressed and never previously-depressed group ("never-depressed"; $n=25$ ). A one-way analysis of variance (ANOVA) with two planned orthogonal contrast analyses was conducted to compare intrusion-based reasoning scores between: 1.) the currently depressed and non-depressed (i.e., the combined previously and never-depressed groups) participants (hypothesis 2) and 2.) the previously-depressed participant group and the never-depressed group (hypothesis 3).

Participant attrition before the follow-up assessment reduced the sample size to the extent that there was insufficient statistical power to compare depressed and non-depressed individuals, or currently, previously and never-depressed participants across the follow-up interval. However, the distributions of BDI (Kolmogorov-Smirnov $\mathrm{Z}=0.76, p=.61$; range 0 to 27), PHQ scores (Kolmogorov-Smirnov $\mathrm{Z}=0.59, p=.88$; range 0 to 20) and BAI scores (Kolmogorov-Smirnov $\mathrm{Z}=0.88, p=.42$; range 0 to 45 ) among the participants who attended the follow-up indicated that anxiety and depressive symptoms formed a continuous distribution. For this reason, we decided to analyse the sample of participants who attended the follow-up assessment as a single unitary sample. 
Results

The majority of the overall sample were female $(n=49 ; 62.8 \%)$, almost half had completed an undergraduate degree $(n=38 ; 48.7 \%)$, and relatively few were taking a psychotropic medication $(n=6 ; 7.7 \%)$ or engaged in psychological therapy at the time $(n=$ $14 ; 17.9 \%)$. A majority of the participants were of Asian $(n=51 ; 65.4 \%)$ or Caucasian $(n=$ $26 ; 33.3 \%)$ background. There were 3 participants who met criteria for a current $\operatorname{PTSD}(n=2$ in the currently-depressed group and $n=1$ in the previously-depressed group). There were no statistically significant differences between the currently-depressed and non-depressed groups on any of the demographic variables (e.g., gender, level of education completed ${ }^{2}$. The selfreport questionnaire and deductive reasoning task scores are summarised in Table 2.

Compared to the non-depressed group, a significantly greater proportion of the depressed group had a co-occurring SCID-derived DSM-IV diagnosis of panic disorder with or without agoraphobia (3.8\% compared with $14.8 \%$ respectively; $\left.\chi^{2}=4.86, d f=1, p=.03\right)$, specific phobia (9.8\% compared with $\left.40.7 \% ; \chi^{2}=10.36, d f=1, p=.001\right)$, and generalised anxiety disorder $\left(9.8 \%\right.$ compared with $\left.29.6 \% ; \chi^{2}=5.00, d f=1, p=.03\right)$. Also, a greater proportion of the depressed group had a history of at least one previous depressive episode (51.0\% compared with $\left.88.9 \% ; \chi^{2}=11.03, d f=1, p=.001\right)$.

\section{Intrusion-based reasoning task}

The internal consistency (Cronbach's alpha) of the two non-self-referent items (how unfortunate and how negative the situation is) was 0.94 for the whole sample. The internal consistency of the four self-referent items (how worthless, incompetent, pathetic and inadequate the situation suggests one is) was also 0.94 . Given these favourable internal consistency values, we report the analyses for non-self-referent and self-referent mean scores respectively ${ }^{1}$. 
Figure 2 shows the intrusion-based reasoning scores for the never-depressed, previously-depressed and currently depressed groups.

Both currently depressed and non-depressed groups appeared to engage in intrusionbased reasoning. The intrusion-based reasoning scores for the depressed group were significantly greater than zero for the self-referent $(t=2.99, d f=26, p=.006)$ and non-selfreferent $(t=4.21, d f=26, p<.001)$ ratings respectively. Likewise, scores for the nondepressed group were significantly greater than zero for the self-referent $(t=6.40, d f=26, p$ $<.001)$ and non-self-referent $(t=6.12, d f=50, p<.001)$ ratings.

When we performed planned comparisons to compare intrusion-based reasoning scores between the currently depressed group and the non-depressed participants (i.e., the combined never and previously-depressed groups), the non-depressed participants scored higher for both non-self-referent (non-depressed $\bar{X}=17.05, S D=20.02$; currently depressed $\bar{X}=14.61, S D=16.95)$ and self-referent ratings (non-depressed $\bar{X}=13.42, S D=15.41$; currently depressed $\bar{X}=10.22, S D=16.96)$, although the differences did not reach statistical significance $(t=-0.76, d f=75, p=.45$ for non-self-referent ratings; $t=-0.85, d f=75, p=.40$ for self-referent ratings) ${ }^{3}$.

Our second planned comparison was between the previously and never-depressed groups. For this analysis, the previously-depressed group scored higher for both self-referent and non-self-referent ratings, although these differences were not statistically significant $(t=$ $1.71, d f=75, p=.09$ for non-self-referent ratings; $t=0.87, d f=75, p=.39$ for self-referent ratings $)^{4}$.

\section{Relationship with mental imagery and deductive reasoning}

When intrusion-based reasoning scores were correlated with SUIS and WST scores, none of the associations reached statistical significance or were greater than \pm .35 in magnitude. 
Six month follow-up

Intrusion-based reasoning scores appeared to remain relatively stable across the sixmonth follow-up interval, as evident by the correlations between intrusion-based reasoning scores at baseline and at follow-up which were typically in the range of 0.4 to 0.6 .The partial correlations between intrusion-based reasoning scores at baseline, and BDI-II scores at follow-up when controlling for baseline BDI-II scores, were not significant.

\section{Discussion}

The results of Study 1 suggest that both dysphoric and non-dysphoric individuals engage in intrusion-based reasoning. In Study 2, our findings were consistent with this in that both the non-depressed and depressed groups demonstrated intrusion-based reasoning, as evidenced by mean intrusion-based reasoning scores that were significantly greater than zero. In this respect, the first hypothesis of Study 2 was confirmed. Whether or not there is a threshold at which intrusion-based reasoning becomes problematic for an individual remains unclear.

A central aim for Study 2 was to determine whether clinically depressed participants engage in intrusion-based-reasoning to a greater extent than non-depressed participants. Contrary to our hypothesis, the currently depressed group did not demonstrate greater levels of intrusion-based reasoning than the non-depressed group. In fact, the depressed group scored non-significantly lower than the non-depressed group for both self- and non-selfreferent intrusion based reasoning. The reasons for this are unclear, although the fact that approximately half of the non-depressed group was comprised of previously-depressed participants - who otherwise scored relatively highly - may have contributed to this.

It is difficult to account for the fact that previously-depressed participants scored nonsignificantly higher than the other two groups. Although we can only speculate, one possible 
reason for this pattern of results may be that previously-depressed individuals may be prone to being vigilant for signs of relapse such as the intensification of intrusive memories. They may also be especially likely to try and "make sense" of these mental events by trying to interpret what the intrusions mean (e.g., "Is this a sign I'm becoming depressed again?"). In contrast, currently depressed individuals may have reached a stage of acceptance regarding their current mental state, and so perceive less need to interpret or make sense of their negative intrusive memories. In any case, the between group differences were not significant and replication of these findings is needed

Despite qualitative differences between self-referent and non-self-referent intrusionbased reasoning, across both studies there were few differences in the patterns of results for each of these. In Study 1, the high dysphoric group scored greater than the low dysphoric group for both non-self-referent and self-referent intrusion-based reasoning, although the difference only reached statistical significance for the self-referent ratings. Likewise, in Study 2 , the pattern of results was the same for both self-referent and non-self-referent intrusionbased reasoning in that the non-depressed group scored non-significantly greater than the depressed group for both non-self-referent and self-referent intrusion-based reasoning. These results then, suggest that intrusion-based reasoning is relied upon for forming a variety of interpretations and that the occurrence of an intrusive memory can colour perceptions of both the self and situations. One possibility is that while self-referent intrusion-based reasoning may play a role in contributing to feelings of worthlessness, low-self-efficacy, and sad mood more generally, non-self-referent intrusion-based reasoning may contribute to the generalisation of sad mood across a variety of situations.

Interestingly, it appears that intrusion-based reasoning is not simply a reflection of dysfunctional attitudes or deficits in general deductive reasoning. Thus, intrusion-based reasoning seems to be independent of the cognitive content that characterises depression (i.e., 
dysfunctional attitudes) as well as the cognitive processes that characterise deficits in deductive reasoning ability. Our impression is that intrusion-based reasoning is a reasoning process whereby mental events are given disproportionate importance in forming conclusions about a situation and about oneself in that situation.

The prospective value of intrusion-based reasoning has not previously been investigated in the absence of trauma. There are two noteworthy aspects of our findings in this regard. First, the strong positive correlations between of intrusion-based reasoning scores at baseline and follow-up suggest that intrusion-based reasoning is a stable tendency. Future studies might investigate the developmental trajectory of intrusion-based reasoning and in which age groups it is most likely to arise. To the best of our knowledge, intrusion-based reasoning has not yet been investigated in samples of children or adolescents. Second, intrusion-based reasoning might not predict subsequent depressive symptoms. When considered alongside the somewhat elevated intrusion-based reasoning scores in our previously-depressed group, our findings suggest that intrusion-based reasoning might not be so much a risk factor for subsequent depressive episodes of depression as it may be a remnant of previous depressive episodes.

It is also noteworthy that the intrusion-based reasoning procedure that we used in each of our studies ensured that there was consistency in the nature of the memory that was retrieved. Previous studies of appraisals of intrusive memories in depression have appropriately relied upon the idiosyncratic memories of participants (e.g., Newby \& Moulds, 2010; Starr \& Moulds, 2006). However, this has also been a limitation in that in such studies, participants provided appraisals of qualitatively different intrusive memories. Additionally, the hypothetical nature of the task (i.e., each participant provided responses to both the presence and the absence of an intrusive memory) allowed us to control for another limitation 
of previous studies, that is, differences between participants in the frequency with which intrusive memories occur.

There are limitations of our studies that call for caution when interpreting the results. Even though the large effect sizes revealed in a previous study of intrusion-based reasoning suggest that we had recruited enough participants to have adequate power, our study lacked sufficient statistical power to detect small size differences between groups. The relatively high degree of variability in participant responses to the intrusion-based reasoning task suggests that improvements to the task itself may increase the power of future studies that use this procedure. Also, despite our efforts to ensure that the scenarios were relevant to most participants, there may have been some participants who were better able to relate to the intrusive memories than others. This may have been particularly likely if some of our participants had experienced the remembered events in their lives but others had not. Unfortunately, we did not ask participants whether they had ever experienced or had an intrusive memory of the events in the intrusion-based reasoning scenarios. Another limitation was that the memories described in our scenarios pertained to negative events. Future studies might investigate intrusion-based reasoning in relation to positively valenced intrusive autobiographical memories and determine the circumstances under which intrusion-based reasoning is problematic. Perhaps depressed individuals are less likely to form negative interpretations when they experience positively toned intrusive memories. Finally, we cannot be sure to what extent the lab-based experimental task used in our studies generalises to real life instances of intrusive memories. There is some evidence to suggest a concordance between lab-based and on-line interpretations of intrusive memories in real life (Williams \& Moulds, 2007c), and use of experience sampling or diary methods may help to answer this question in future. 
Previous studies of intrusion-based reasoning have focused on PTSD. While the findings of these studies demonstrate that intrusion-based reasoning characterises PTSD (Engelhard et al., 2001; Engelhard et al., 2002), our studies suggest that its association with depression is less clear. Specifically, in PTSD, intrusion-based reasoning is not only characteristic of PTSD (Engelhard et al., 2001), but the presence of this reasoning process has prospective value in predicting who will develop PTSD symptoms (Engelhard et al., 2002). This seems not to be the case for depression, in which (i) intrusion-based reasoning might serve as a better indicator of previous episodes than a current episode, and (ii) the presence of intrusion-based reasoning may not predict subsequent symptoms or depressive episodes.

Together, these two studies suggest that although self-referent intrusion-based reasoning appears to be elevated in dysphoric individuals, neither type of intrusion-based (i.e., self- or non-self referent) reasoning is especially prominent in clinical depression. Instead, intrusion-based reasoning may be a marker of previous depressive episodes, although our results in this regard were not significant. Finally, intrusion-based reasoning appears to be a stable tendency, but not necessarily predictive of subsequent depressive symptoms. 


\section{Footnotes}

1. The results for each of the individual ratings are available from the authors upon request.

2. We compared the ethnic compositions of the three participant groups (i.e., currently depressed, previously-depressed and never-depressed). When the proportion of Asian and Caucasian participants in the never-depressed group was compared with the currently depressed group, there were proportionately more Asian participants in the never-depressed group $\left(\chi^{2}=4.03, d f=1, p=0.045\right)$. However, importantly, there were no significant differences in intrusion-based reasoning scores according to ethnicity.

3. Given that the elevated intrusion-based reasoning scores for the non-depressed group may have been contributed to by inclusion of previously-depressed participants, we conducted a post-hoc comparison between the currently depressed and neverdepressed groups. When we did this, the currently depressed group scored marginally higher than the never-depressed group for non-self-referent ratings, and marginally lower than the never-depressed group for self-referent ratings, although these differences were not statistically significant (all $p \mathrm{~s}>.05$ ).

4. Given that inspection of Figure 2 suggests that the previously-depressed group appeared to score higher for both forms of intrusion-based reasoning than the currently depressed group, we also conducted a post-hoc comparison of the previously- and currently depressed groups. When we did this, there were no significant differences between the previously- and currently depressed groups (all $p$ s $>.05)$ on either self-referent or non-self-referent intrusion-based reasoning. 


\section{References}

Beck, A.T., Rush, A.J., Shaw, B.F., \& Emery G (1979). Cognitive Therapy of Depression. New York: Guilford Press.

Beck, A. T., Steer, R. A., \& Brown, R. A. (1996). Beck Depression Inventory, 2nd Edition. San Antonio: The Psychological Corporation, Harcourt Brace \& Company.

Berntsen, D. (2010). The unbidden past. Current Directions in Psychological Science, 19, 138-142. doi: $10.1177 / 0963721410370301$

Berle, D. \& Moulds, M. L. (in press). An experimental investigation of emotional reasoning processes in depression. British Journal of Clinical Psychology. doi: 10.1111/bjc. 12019

Birrer, E., Michael, T., \& Munsch, S. (2007). Intrusive images in PTSD and in traumatised and non-traumatised depressed patients: a cross sectional clinical study. Behaviour Research and Therapy, 45, 2053-2065. doi: 10.1016/j.brat.2007.03.005

Brewin, C. R., Reynolds, M., Tata, P. (1999). Autobiographical memory processes and the course of depression. Journal of Abnormal Psychology, 108, 511-517. doi: $\underline{10.1037 / 0021-843 X .108 .3 .511}$

Dunmore, E., Clark, D. M., \& Ehlers, A. (2001). A prospective investigation of the role of cognitive factors in persistent posttraumatic stress disorder (PTSD) after physical or sexual assault. Behaviour Research and Therapy, 39, 1063-1084. doi: 10.1016/S00057967(00)00088-7

Engelhard, I. M., Macklin, M. L., McNally, R. J., van den Hout, M. A., \& Arntz, A. (2001). Emotion- and Intrusion-based reasoning in Vietnam veterans with and without chronic posttraumatic stress disorder. Behaviour Research and Therapy, 39, 12291348. doi: $\underline{10.1016 / \mathrm{S} 0005-7967(00) 00101-7}$

Engelhard, I. M ., van den Hout, M. A., Arntz, A., \& McNally, R. J. (2002). A longitudinal study of "intrusion-based reasoning" and posttraumatic stress disorder after exposure 
to a train disaster. Behaviour Research and Therapy, 40, 1415-1424. doi:

$10.1016 / \mathrm{S} 0005-7967 \% 2802 \% 2900018-9$

First, M. B., Spitzer, R. L., Gibbon, M., \& Williams, J. B. W. (1996). Structured Clinical Interview for DSM-IV Axis I Disorders (SCID-I). American Psychiatric Press, Washington, DC.

Harvey, A. G., Watkins, E., Mansell, W., \& Shafran, R. (2004). Cognitive behavioural processes across psychological disorders: A transdiagnostic approach to research and treatment. Oxford, UK: Oxford University Press.

Kosslyn, S. M., Chabris, C. F., Shephard, J. M., \& Thompson, W. L. (1998). Spontaneous Use of Imagery Scale (SUIS). Unpublished manuscript.

Kovacs, M., \& Beck, A. T. (1978) Maladaptive cognitive structures in depression. American Journal of Psychiatry, 135, 525-533.

Moulds, M. L., \& Holmes, E. A. (2011). Intrusive Imagery in Psychopathology: A Commentary. International Journal of Cognitive Therapy, 4, 197-207. doi: 10.1521/ijct.2011.4.2.197

Nelson, L. D., Stern, S. L., \& Cicchetti, D. V. (1992). The Dysfunctional Attitude Scale: How well can it measure depressive thinking? Journal of Psychopathology and Behavioral Assessment, 14, 217-223. doi: 10.1007/BF00962629

Newby, J. M., \& Moulds, M. L.(2010). Negative intrusive memories in depression: The role of maladaptive appraisals and safety behaviours. Journal of Affective Disorders, 126, 147-154. doi: 10.1016/j.jad.2010.03.012

Newby, J. M., \& Moulds, M. L. (2011a). Characteristics of intrusive memories in a community sample of depressed, recovered depressed and never-depressed individuals. Behaviour Research and Therapy, 49, 234-243. doi: 10.1016/j.brat.2011.01.003 
Newby, J. M. \& Moulds, M. L. (2011b). Do negative appraisals and avoidance of intrusive memories predict depression at six months? International Journal of Cognitive Therapy, 4, 178-186. doi: 10.1521/ijct.2011.4.2.178

Nolen-Hoeksema, S. (1991). Responses to depression and their effects on the duration of depressive episodes. Journal of Abnormal Psychology, 100, 569-582. doi: $10.1037 / 0021-843 X .100 .4 .569$

Rachman, S. (1981). Part 1: unwanted intrusive cognitions. Advances in Behaviour Research and Therapy, 3, 89-99. doi: 10.1016/0146-6402(81)90007-2

Rachman, S., \& de Silva, P. (1978). Abnormal and normal obsessions. Behaviour Research and Therapy, 16, 233-248. doi: 10.1016/0005-7967(78)90022-0

Reisberg, D., Pearson, D. G., \& Kosslyn, S. M. (2003). Intuitions and introspections about imagery: The role of imagery experience in shaping an investigator's theoretical views. Applied Cognitive Psychology, 17, 147-160. doi: 10.1002/acp.858

Segal, Z. V., Kennedy, S., Gemar, M., Hood, K., Pedersen, R., \& Buis, T. (2006). Cognitive reactivity to sad mood provocation and the prediction of depressive relapse. Archives of General Psychiatry, 63, 749-755. doi: 10.1001/archpsyc.63.7.749

Starr, S., \& Moulds, M. L. (2006). The role of negative interpretations of intrusive memories in depression. Journal of Affective Disorders, 93, 125-132. doi: 10.1016/j.jad.2006.03.001

Wason, P. C. (1968). Reasoning about a rule. Quarterly Journal of Experimental Psychology, 20, 273-281. doi: 10.1080/14640746808400161

Weissman AN, \& Beck AT (1978) Development and validation of the dysfunctional attitude scale. Paper presented at the Annual Meeting of the Association for the Advancement of Behavior Therapy, Chicago, November. 
Williams, A. D., \& Moulds, M. L. (2007a). Cognitive avoidance of intrusive memories: recall vantage perspective and associations with depression. Behaviour Research and Therapy, 45, 1141-1153. doi: 10.1016/j.brat.2006.09.005

Williams, A. D., \& Moulds, M. L. (2007b). Investigation of the indulgence cycles hypothesis of suppression on experimentally induced visual intrusions in dysphoria. Behaviour Research and Therapy, 45, 2780-2788. doi: 10.1016/j.brat.2007.07.001

Williams, A. D., \& Moulds, M. L. (2007c). An investigation of the cognitive and experiential features of intrusive memories in depression. Memory, 15, 912-920. doi: $10.1080 / 09658210701508369$

Williams, A. D., \& Moulds, M. L. (2008). Negative appraisals and cognitive avoidance of intrusive memories in depression: A replication and extension. Depression and Anxiety, 25, E26-E33. doi: 10.1002/da.20409

Wisco, B. E. (2009). Depressive cognition: Self-reference and depth of processing. Clinical Psychology Review, 29, 382-392. doi: 10.1016/j.cpr.2009.03.003

Wisco, B. E., \& Nolen-Hoeksema, S. (2010). Interpretation bias and depressive symptoms: The role of self-relevance. Behaviour Research and Therapy, 48, 1113-1122. doi: 10.1016/j.brat.2010.08.004 


\section{Acknowledgements}

The authors are grateful to Prof. Gavan McNally for suggestions regarding the deductive reasoning task and to Emily Phillips for her assistance in co-rating the diagnostic interviews. Michelle Moulds is supported by an Australian Research Fellowship from the Australian Research Council (ARC). 
Table 1. Demographic variables and self-report questionnaire scores for the low dysphoric, high dysphoric and total sample for Study 1.

\begin{tabular}{|c|c|c|c|c|c|c|c|c|}
\hline & \multicolumn{2}{|c|}{$\begin{array}{c}\text { Low dysphoric } \\
\quad(n=28)\end{array}$} & \multicolumn{2}{|c|}{$\begin{array}{l}\text { High dysphoric } \\
\quad(n=42)\end{array}$} & \multicolumn{2}{|c|}{$\begin{array}{l}\text { Total sample } \\
(N=70)\end{array}$} & \multicolumn{2}{|c|}{$\begin{array}{l}\text { Comparing } \\
\text { proportion of } \\
\text { females in Low } \\
\text { vs High } \\
\text { dysphoric } \\
\text { groups }\end{array}$} \\
\hline & $n$ & $\%$ & $n$ & $\%$ & $n$ & $\%$ & $\chi^{2}$ & $\begin{array}{c}p- \\
\text { value }\end{array}$ \\
\hline \multirow[t]{3}{*}{ Females } & 14 & 50.00 & 28 & 66.67 & 42 & 60.00 & 1.94 & 0.16 \\
\hline & & & & & & & \multicolumn{2}{|c|}{$\begin{array}{l}\text { 95\% Confidence } \\
\text { Interval for Low } \\
\text { vs High } \\
\text { dysphoric } \\
\text { groups }\end{array}$} \\
\hline & Mean & SD & Mean & SD & Mean & SD & Lower & Upper \\
\hline Age & 18.82 & 1.22 & 19.26 & 2.58 & 19.09 & 2.14 & -1.49 & 0.60 \\
\hline
\end{tabular}

Self-report questionnaire:

\begin{tabular}{lllllllll} 
DAS & 104.82 & 20.50 & 140.81 & 32.52 & 126.41 & 33.29 & -49.80 & -22.18 \\
\hline DAS
\end{tabular}

DAS = Dysfunctional Attitudes Scale - Form A. 
Figure 1. Intrusion based reasoning scores for low and high dysphoric groups in Study 1.

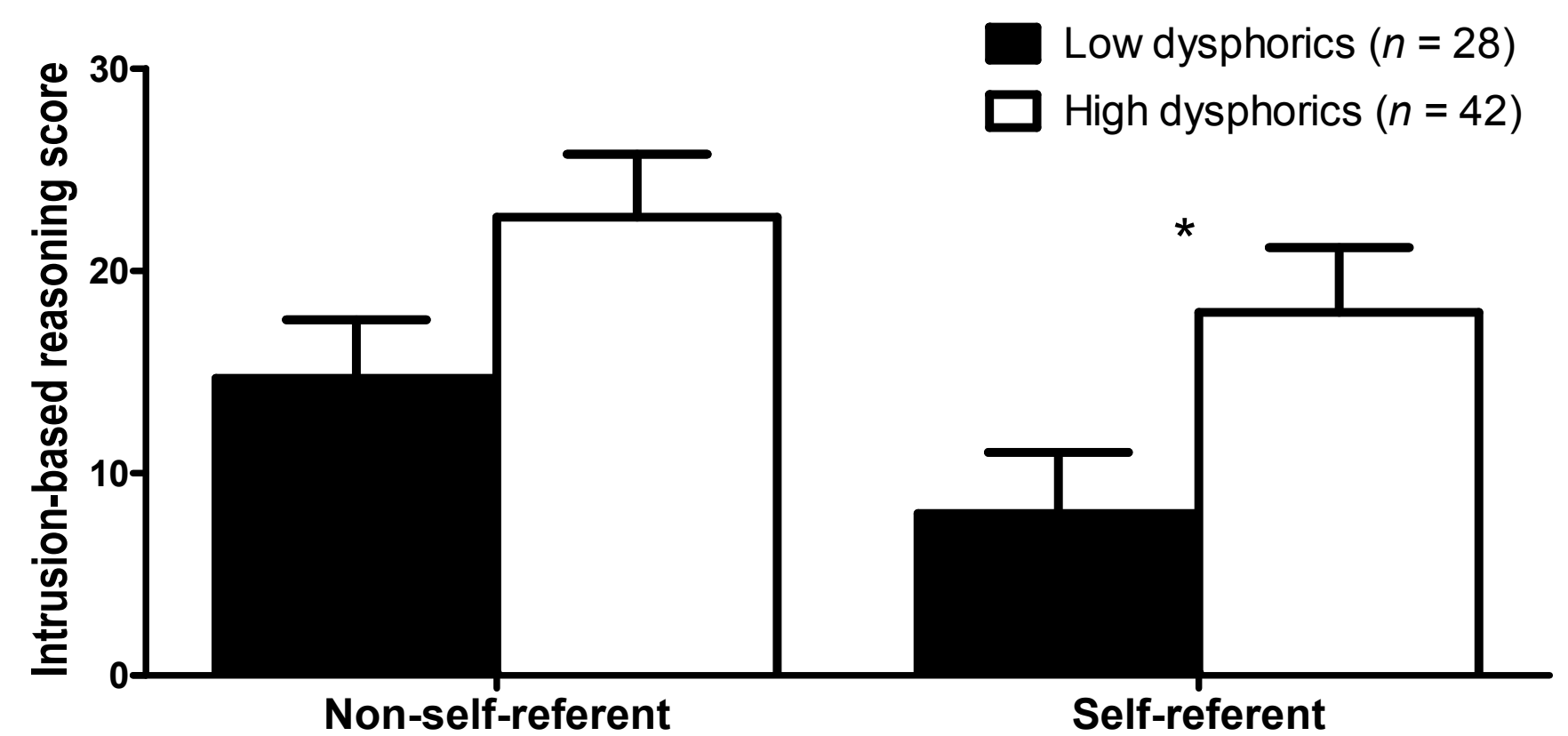

${ }^{*} p<.05$ for difference between high and low dysphoric groups. Error bars are the standard error of the mean. 
Table 2. Self-report scores, deductive reasoning scores and rates of co-occurring PTSD in Study 2.

\begin{tabular}{|c|c|c|c|c|c|c|c|c|c|}
\hline & \multicolumn{2}{|c|}{$\begin{array}{c}\text { Never-depressed } \\
\quad(n=25)\end{array}$} & \multicolumn{2}{|c|}{$\begin{array}{c}\text { Previously- } \\
\text { depressed } \\
(n=26)\end{array}$} & \multicolumn{2}{|c|}{$\begin{array}{c}\text { Currently } \\
\text { depressed } \\
(n=27)\end{array}$} & \multicolumn{3}{|c|}{$\begin{array}{l}\text { Total sample } \\
(N=78)\end{array}$} \\
\hline & Mean & $S D$ & Mean & $S D$ & Mean & $S D$ & Mean & $S D$ & Cronbach's $\alpha$ \\
\hline \multicolumn{10}{|c|}{ Depression symptom self-report questionnaires: } \\
\hline BDI-II & 6.96 & 5.74 & 9.50 & 6.30 & 19.56 & 4.65 & 12.17 & 7.80 & .92 \\
\hline PHQ-9 & 4.32 & 3.91 & 6.77 & 5.62 & 14.78 & 4.56 & 8.76 & 6.52 & .91 \\
\hline \multicolumn{10}{|c|}{ Other self-report questionnaires: } \\
\hline BAI & 31.64 & 8.40 & 32.46 & 10.01 & 44.15 & 12.33 & 36.24 & 11.81 & .93 \\
\hline SUIS & 36.76 & 7.07 & 40.65 & 9.80 & 39.04 & 9.05 & 38.85 & 8.77 & .82 \\
\hline \multicolumn{10}{|l|}{ Reasoning task: } \\
\hline $\begin{array}{l}\text { Wason Selection Task } \\
\text { total score* }\end{array}$ & 9.56 & 2.35 & 10.12 & 2.39 & 9.52 & 2.81 & 9.73 & 2.51 & \\
\hline
\end{tabular}

total score*

* Wason selection task total score calculated as the sum of verification and falsification scores.

BAI = Beck Anxiety Inventory; BDI-II = Beck Depression Inventory II; CERQ = Cognitive Emotion Regulation Questionnaire; ERQ = Emotion Regulation Questionnaire; PHQ = Patient Health Questionnaire; SUIS = Spontaneous Use of Imagery Scale. 
Figure 2. Intrusion-based reasoning scores for the never-depressed, previously-depressed and currently depressed groups in Study 2.

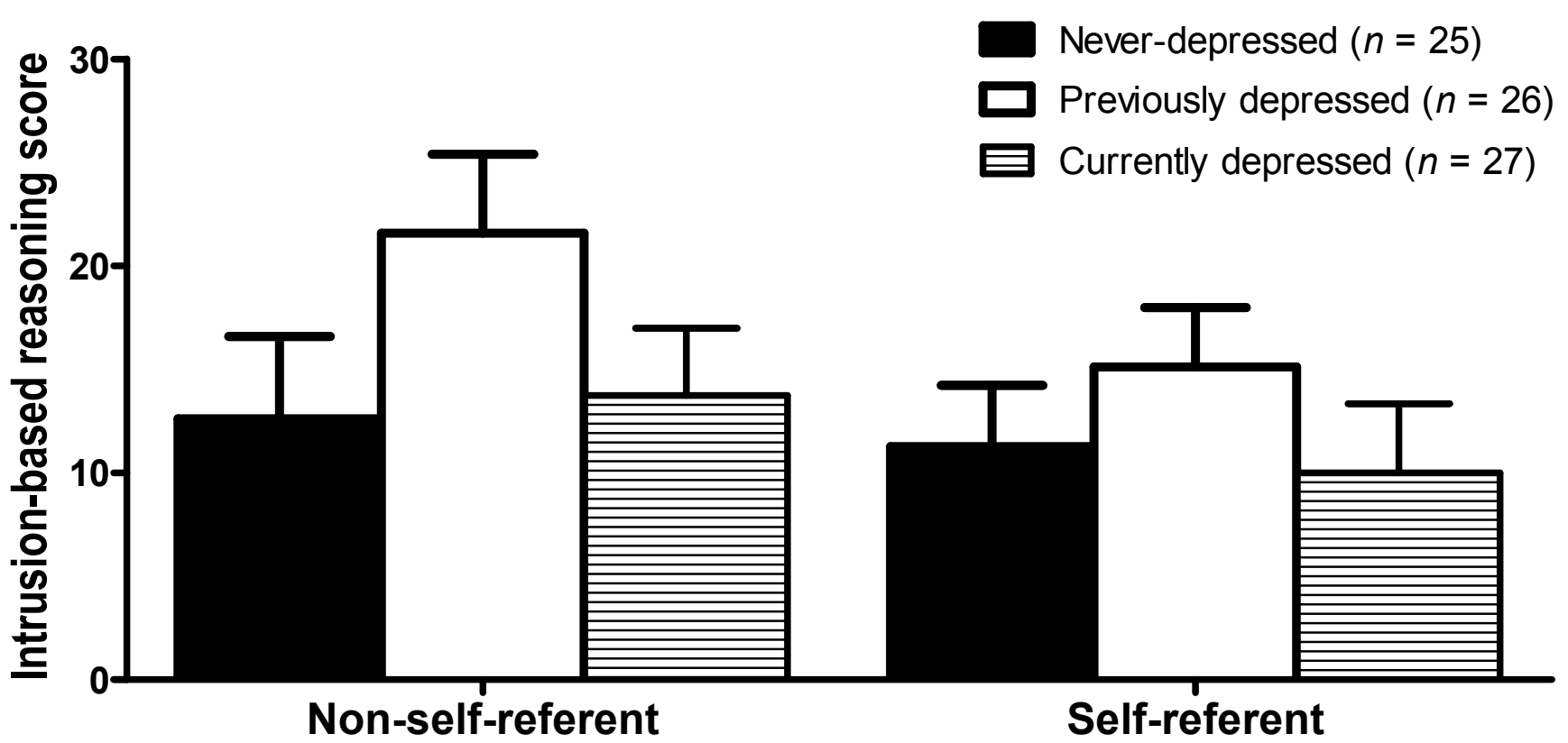

Error bars are the standard error of the mean. 